\title{
On the Structural Differences between Disk and Dwarf Galaxies
}

\author{
James M. Schombert \\ Department of Physics, University of Oregon, Eugene, OR 97403; js@abyss.uoregon.edu
}

\begin{abstract}
Gas-rich dwarf and disk galaxies overlap in numerous physical quantities that make their classification subjective. We report the discovery of a separation between dwarfs and disks into two unique sequences in the mass (luminosity) versus scale length plane. This provides an objective classification scheme for late-type galaxies that only requires optical or near-IR surface photometry of a galaxy. Since the baryonic Tully-Fisher relation for these samples produces a continuous relation between baryonic mass and rotational velocity, we conclude that the difference between dwarfs and disks must be because of their distribution of stellar light such that dwarfs are more diffuse than disk galaxies. This structural separation may be due to a primordial difference between low and high mass galaxies or produced by hierarchical mergers where disks are built up from dwarfs. Structural differences between dwarf and disk galaxies may also be driven by the underlying kinematics where the strong rotation in disks produces an axial symmetric object that undergoes highly efficient star formation in contrast to the lower rotation, more disordered motion of dwarfs that produces a diffuse, triaxial object with a history of inefficient star formation.
\end{abstract}

Subject headings: galaxies: dwarf, galaxies: formation, galaxies: structure

\section{INTRODUCTION}

Gas-rich galaxies dominate the far end of the Hubble scheme and are primarily composed of two types, dwarf irregulars and spirals disks. While differing in general morphological appearance, the latetype dwarfs and disks also separate in terms of mean luminosity and size. However, these galaxies are not uniquely determined by their mass or size as there exist many examples of massive irregulars (Hunter \& Gallagher 1986) as well as dwarf spirals (Patterson \& Thuan 1996, Schombert et al. 1995). To first order, their kinematics are similar, both being dominated by rotation (van Zee, Salzer \& Skillman 2001) and their star formation histories are active both today and in the recent past (McGaugh \& de Blok 1997).

A connection between dwarfs and disks is also inferred by the baryonic Tully-Fisher relation (McGaugh et al. 2000). The constant slope and low scatter of dwarfs and disks in the mass-rotational velocity plane implies that the corrections from luminosity to mass (IMF, $M / L$, gas content) are similar from dwarfs to disks and that the total baryonic mass is directly proportional to the total mass. So, while the dark matter fraction may be higher in dwarfs compared to spirals, this difference scales evenly across the Hubble sequence. This has implications for galaxy formation scenarios as hierarchical models, which would not necessary predict two families of galaxies, would be supported by the discovery of distinct differences in the properties of low and high mass galaxies (Kauffmann et al. 2003). 
Given their similarities, the operative question becomes is there any difference between disks and dwarfs or are they simply a continuous sequence where some underlying physical parameter (such as angular momentum or gas fraction) determines the size and appearance of a gas-rich galaxy. The goal of this paper is to compare the properties of dwarf and disk galaxies by fitting their surface brightness profiles in order to extract total luminosities, central surface brightnesses and scale lengths in the search of structural properties which may separate disk galaxies from dwarf irregulars. We will also examine the H I properties of the two samples (such as line profile shape and gas fraction) for details concerning kinematics and star formation history that may suggest different paths of evolution between dwarfs and disks.

\section{ANALYSIS}

The data for this paper derives from three studies on the optical and H I properties of dwarf and disk galaxies. For the disk samples, we have used the data on ordinary spirals extracted from two surveys, Courteau (1996) and de Jong (1996). The Courteau survey was primarily focused on Sc galaxies, as probes to the Tully-Fisher relation, and presented CCD and H I observations of 189 galaxies. The de Jong sample contains a range of spiral types selected from the UGC with diameters greater than two arcmins and undisturbed in their morphology, a total of 62 galaxies. The dwarf sample of 107 galaxies is extracted from the LSB catalogs of Schombert et al. (1997). Both the optical and H I properties of these three samples were discussed in detail in Schombert, McGaugh \& Eder (2001). That paper focused on the gas mass fractions of disk and dwarf galaxies in order to understand their star formation histories and color evolution. This paper will focus solely on the integrated optical and H I properties of the three samples as well as their structural properties as given by surface photometry. All distance related quantities in this paper use values of $H_{o}=75 \mathrm{~km} \mathrm{sec}^{-1} \mathrm{Mpc}^{-1}$, a Virgo central velocity of 977 $\mathrm{km} \mathrm{sec}^{-1}$ and a Virgo infall of $300 \mathrm{~km} \mathrm{sec}^{-1}$. The data used for this study is available at the LSB dwarf web site (abyss.uoregon.edu/ js).

The structural properties for both the disk and dwarf samples are determined by exponential fits to their surface brightness profiles. An exponential light profile is an almost universal observational feature of disk galaxies (de Jong 1996) parameterized by two quantities, the central surface brightness $\left(\mu_{o}\right)$ and the disk scale length $(\alpha)$. Likewise, the galaxies in the LSB dwarf sample are also well fit by an exponential light profile (Pildis, Schombert \& Eder 1997), although there is no assumption that the shape of the underlying mass distribution is identical to spirals (see section §2.1). From these fits, the total luminosity of the galaxy (integrated light based on elliptical apertures from the surface

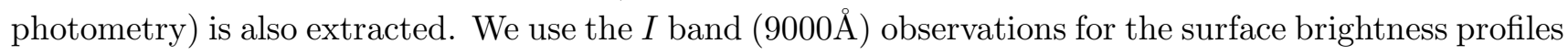
since the far-red provides a better measure of the total stellar mass compared to near-blue colors.

In studying structure of a galaxy, as given by its surface brightness profile, one is less interested in the luminosity of a galaxy than its mass. Gas masses can be determined directly from 21-cm fluxes (with a small correction for the contribution from molecular gas). However, the conversion of luminosity to mass requires knowledge of the mean $M / L$ of the underlying stellar population. As discussed in Schombert, McGaugh \& Eder (2001), we follow the prescription of McGaugh et al. (2000) and adopt a value of $M / L=1.7$ to convert luminosities into solar masses. The structural and mass properties of the three samples are summarized in Figures 1 and 2, plots of galaxy mass versus central surface brightness 


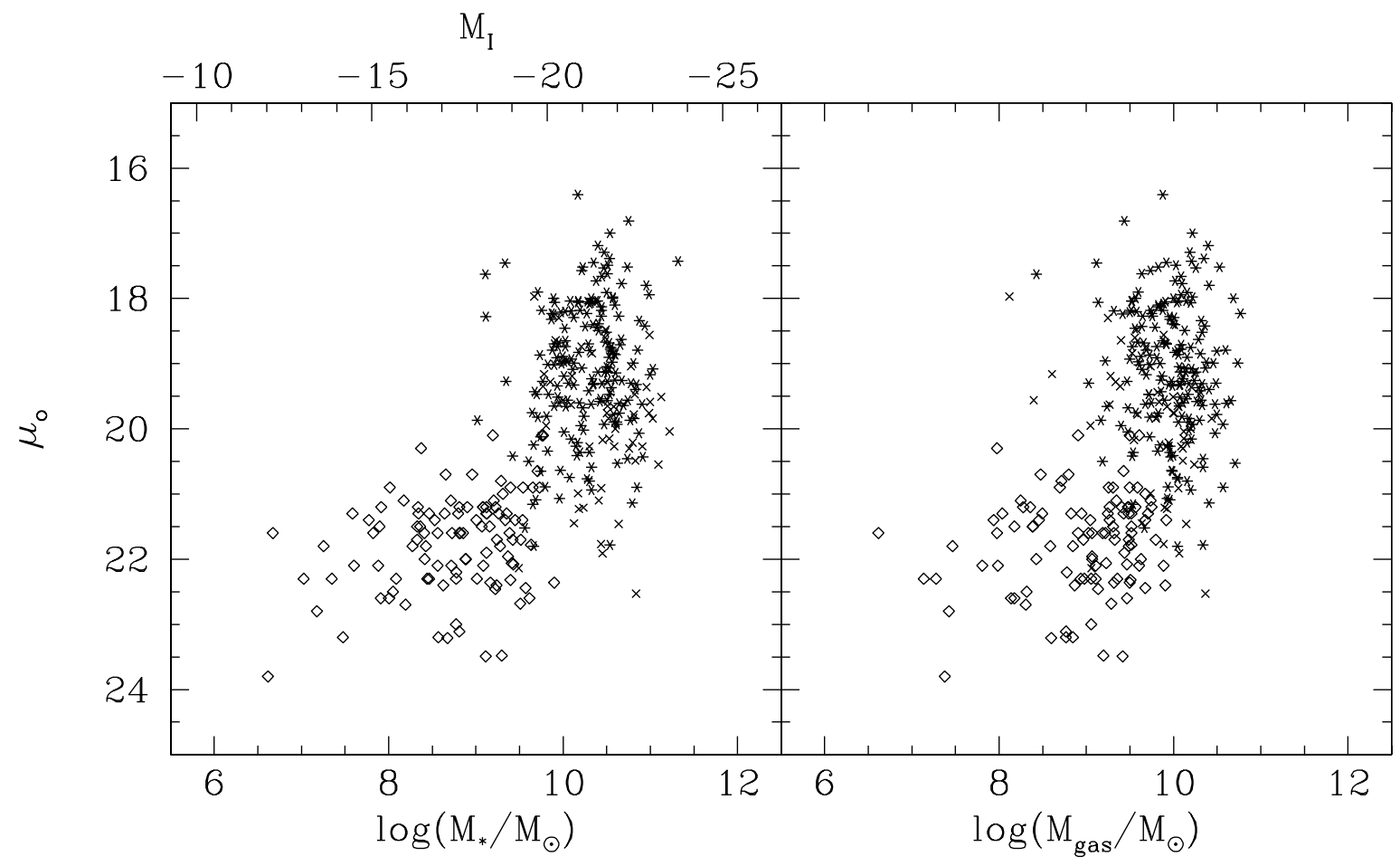

Fig. 1. - Stellar and gas mass versus central optical surface brightness $\left(\mu_{o}\right)$. The open symbols are from the LSB dwarf catalog, crosses are disks from de Jong (1996) and asterisks are Sc galaxies from Courteau (1996). Luminosity is converted to stellar mass using an $M / L$ of 1.7 (see text). Gas mass is determined from the integrated HI flux corrected for molecular gas. The selection criteria for the LSB dwarf sample was low in mean surface brightness and irregular in morphology. This will naturally produce a sample of low central surface brightness, low mass objects which occupy the lower lefthand portion of the diagram. Disk galaxies, having higher rates of past star formation, occupy the upper portion of the diagram. Stability requirements keep their masses above those of dwarfs. 
$\left(\mu_{o}\right)$ and scale length $(\alpha)$. The galaxy mass is divided into two parts, the mass of stars $\left(M_{*}\right)$ as given by the $I$ band luminosity and the mass in gas $\left(M_{\text {gas }}\right)$ as deduced from the H I observations. The sum of these two represents the total baryonic mass of a galaxy (McGaugh et al. 2000).

Figure 1 displays the effect of a morphological selection combined with low surface brightness criteria to the LSB dwarfs. Both of the disk galaxy samples display a larger range of central surface brightnesses than the dwarf sample reflecting their typically higher past rates of star formation. The disk samples also have higher mean masses as required by disk stability requirements (Fall \& Efstathiou 1980). While there is a trend of lower central surface brightness with decreasing mass, this is, in part, due to how the samples were selected. The LSB dwarf sample was intentionally selected to be of lower surface brightness than objects in the UGC catalog. With the additional constraint of irregular morphology (Schombert, Pildis \& Eder 1997), this criteria produces a sample of galaxies with low baryonic mass and low $\mu_{o}$. The upper left corner of Figure 1 would be populated by blue compact dwarfs (BCD's, see Patterson \& Thuan 1996) and dwarf ellipticals (dE's), but these types of galaxies are not found in any of the three samples used herein. There is no strict linear correlation in Figure 1 and galaxy formation models predict galaxies will occupy all regions of this diagram (Dalcanton, Spergel \& Summers 1997) even if found to be rare in our galaxy catalogs. The diagram is more compact in the right hand panel for gas mass simply because of the gas-rich nature of dwarf irregulars (i.e. more gas than stars).

Figure 2 displays the relationship between galaxy mass and scale length. All three sets of galaxies span five orders of magnitude in stellar mass and a factor of 50 in scale length. The obvious correlation is evident, i.e. larger galaxies are more massive. However, it is also clear from left-hand panel of Figure 2 that the data (in terms of stellar mass versus scale length) form two separate and distinct groups. One group is delineated primarily by the LSB dwarfs and lies above (larger $\alpha$ ) and to the left (lower stellar mass) of the second group outlined by the disk samples. While there are a few disk galaxies located in the LSB dwarf group, a majority of those galaxies are Sm class (see below).

Each group in Figure 2 is best described by a linear sequence. The two sequences, which we will refer to as the dwarf and disk sequences, are not a single track with a changing slope and there is no evidence of any deviation from a straight line for each sample (see fits below). There appears to be a break at $M_{*}=10^{10} M_{\odot}$, but closer inspection shows that there are several galaxies on the dwarf track of higher mass, and several spirals on the disk track lower in mass but still following their respective sequences. A biweight fit to the dwarf sample results in the following relation:

$$
\log \alpha=0.40 \pm 0.02 \log M_{*} / M_{\odot}-3.37 \pm 0.15[\text { dwarfs }]
$$

A biweight fit solely to the disk samples results in:

$$
\log \alpha=0.44 \pm 0.02 \log M_{*} / M_{\odot}-3.96 \pm 0.19[\text { disks }]
$$

where both fits are shown in Figure 2 as dashed lines. Inspection of the dwarf galaxy data finds all the dwarfs to lie above the disk relation. This cannot be due to a change in slope for the dwarf sample as the linear fit to the dwarfs is statistically the same slope as the disk sample. The scatter around the linear fits is similar for the dwarf and disk samples, even though the disk sample represents a brighter and photometrically more accurate dataset. This is probably a statement concerning the meaning of 


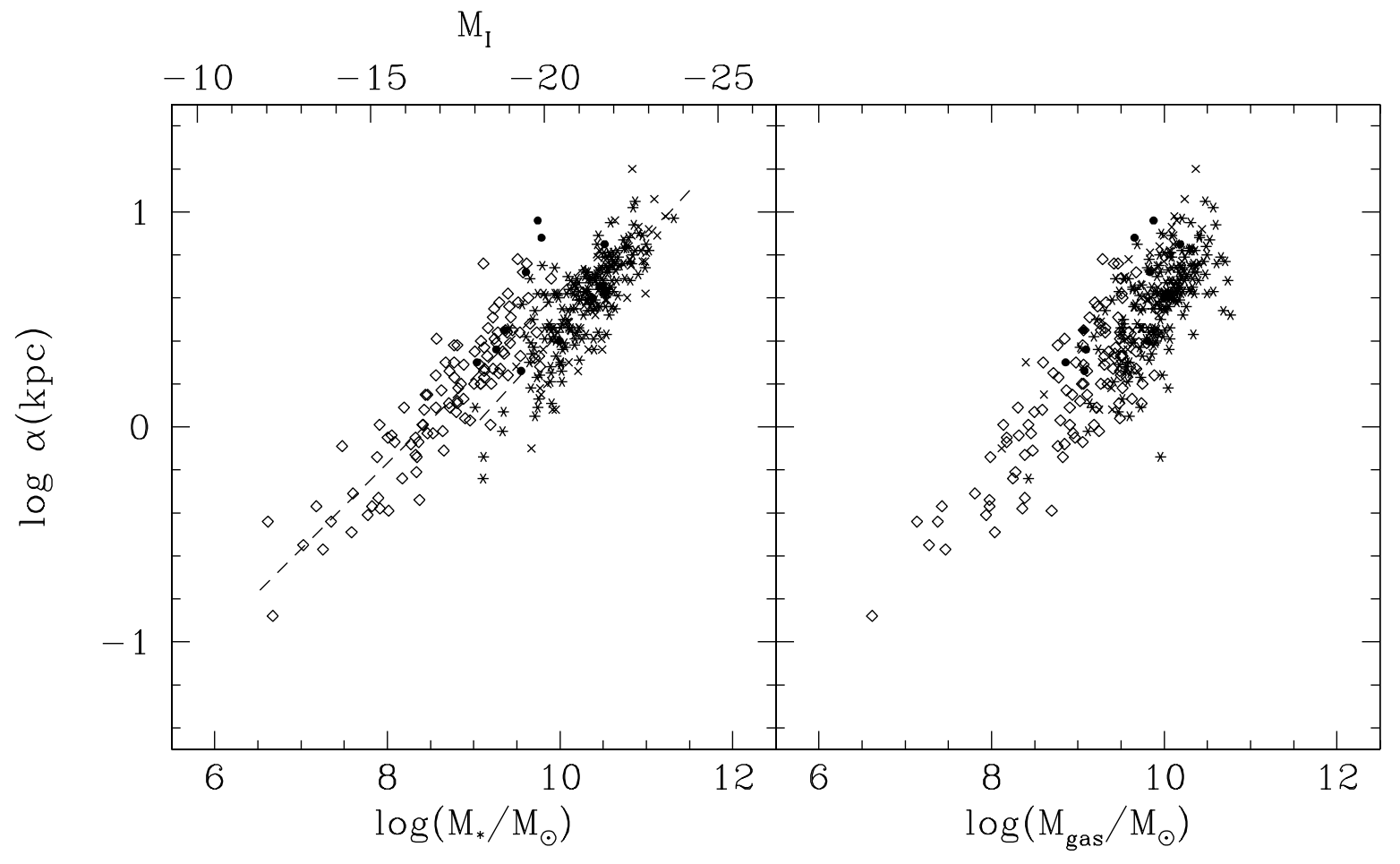

Fig. 2.- Stellar and gas mass versus optical scale length $(\alpha)$ in kpc. The open symbols are from the LSB dwarf catalog, crosses are disks from de Jong (1996) and asterisks are Sc galaxies from Courteau (1996). The separation of dwarfs and disks into two sequences is evident in the left panel. Sm class galaxies from de Jong are shown as solid symbols and are typically found on the dwarf sequence. Biweight fits to each sample is shown as dashed lines. 
the parameters extracted from surface photometry fits. While both dwarfs and disks are well fit by an exponential for some portion of their surface brightness profiles, the existence of bulges and irregular luminosities distributions will reflect as variations in the total magnitude as compared to a structural parameter such as scale length $(\alpha)$ in Figure 2. A disk or integrated magnitude based on the surface brightness profiles produces less scatter, but than fails to reflect the total stellar mass of a galaxy (see Graham \& Driver 2005). There is no indication that the scatter in Figure 2 disguises a non-linear correlation between $\alpha$ and stellar mass, and the mean scatter is less than the separation of the two dwarf and disk sequences. When examining the distributions of the disk and dwarf data around the linear fits, and using a $t$-test to compare the resulting histograms, we find that we must reject the hypothesis that the two samples derive from a common population at the $99.99 \%$ confidence level.

The raw interpretation for Figure 2 is that LSB dwarf galaxies either have large scale lengths for their stellar mass, compared to disks, or they have less stellar mass compared to disks of the same scale length (i.e. they are too big for their mass or under mass for their size). The slopes of the two sequences are statistically indistinguishable from a value of 0.4 . We note that this produces a relationship of $L \propto \alpha^{2}$ and, therefore, given that the total luminosity of an exponential profile is $L=2 \pi \alpha^{2} \Sigma_{o}$, this implies that the optical luminosity of a galaxy will be independent of their surface brightness (see Hoffman et al. 1996). This is certainly true of LSB galaxies, where a range of luminosities, masses and sizes is found from the LSB dwarfs to the Malin giants.

While the relationship between stellar mass and scale length $(\alpha)$ differs between disks and dwarfs, the same is not true with respect to gas mass. The right panel in Figure 2 displays the relationship for gas mass, and the two samples overlap into one continuous population. This is due, primarily, to the differences in gas content between spiral disks and LSB dwarfs. LSB dwarfs have more of their baryonic mass in the form of gas compared to disks. Thus, the disk sample decreases in the ratio of gas mass to stellar mass compared to dwarfs and the two samples converge.

An interesting morphological point, there are several disks from the de Jong study on the dwarf track, but defined as disks based on their morphological appearance (solid symbols in Figure 2). All of these galaxies are Sm class, whereas the spirals on the disk track are classed as Sd or earlier (see §2.1). None of the Courteau Sc galaxies appear on the dwarf sequence. Previously, Patterson \& Thuan (1996) have shown that dwarf spirals link, structurally, to LSB and ordinary disks. The inclusion of these objects would extend the disk sequence to approximately $M_{T}=-17$ or stellar masses of $5 \times 10^{8} M_{\odot}$.

Why this separation of dwarfs and disks in the $M_{*}-\alpha$ diagram has gone unnoticed is not clear. The sequence is obvious in Patterson \& Thuan's data (see their Figure 10); however, their interpretation is focused on the difference between gas-rich dwarfs (dI), dwarf ellipticals (dE) and blue compact dwarfs (BCD), and the possible evolutionary connections between those types. In fact, the dwarf ellipticals appear to form a third sequence, fainter for their size compared to spirals (or larger for their luminosity). The two sequences are also visible in Figure 2 of McGaugh \& de Blok (1997), and they even comment on the discrepancy for the $\mathrm{Sm} / \mathrm{Im}$ galaxies in the sample from the relationship in the $L_{B}-\alpha$ plane for Sa through Sd type galaxies. Perhaps one reason that this separation is more obvious in Figure 2 is that this study introduces another 5 magnitudes of dynamic range for the dwarf sequence as compared to previous work and surveys strictly LSB galaxies. HSB type dwarfs, such as BCD's, appear to be related to dE's (Papaderos et al. 1996) and blur the relationship as they lie on the disk sequence. 
One explanation for the separation of dwarfs and disks in the $M_{*}-\alpha$ diagram would be an error in the conversion from light to mass (i.e. the assumed $M / L$ ). In order to bring the two sequences into alignment, an error in $M / L$ between dwarfs and disks is required in the direction that the dwarfs must have higher $M / L$ 's by a factor of 8 . This is not plausible for a number of reasons. First, the luminosities selected to convert to mass are $I$ band (far-red) measurements. Stellar population models (Worthey 1994) demonstrate that $M_{*} / L$ varies with time and star formation rate as a function of wavelength, but is most stable in the far-red because of its distance in wavelength from the region around the $4000 \AA$ break. Thus, $I$ band measurements provide a more accurate estimate of the stellar mass of a galaxy and obtain a luminosity measure that vary little with recent star formation. Second, this effect was examined in detail in McGaugh \& de Blok (1997) who concluded that LSB galaxies cannot vary by more than $20 \%$ in $M / L$ as adjusted for color effects. Lastly, a study by Bell \& de Jong (2001) indicates that $M / L$ can vary by as much as a factor of two over the color range of LSB dwarfs, but this is still well short of the factor of 8 required to align the dwarf and disk sequences in Figure 2.

Another possible explanation to consider is a mismatch in the meaning of scale length from disk to dwarfs. The exponential profile is a good fit to rotating disks for kinematic reasons. But the justification for an exponential profile in dwarf irregular galaxies is solely based on the empirical quality of the fit to the surface brightness profiles of dwarfs (Patterson \& Thuan 1996). This is particularly true in the $I$ band where structural parameters (such as scale length) are undistorted by recent star formation events or dust and provide a smoother measure of the underlying stellar mass distribution. However, one concern is that $\alpha$ is measuring some quantity in 3D space that is not reflected into the sky distribution in the same manner between dwarfs and disks. This, then, concerns the true shape of dwarfs and disks.

It was concluded from imaging work that disk galaxies are, in general, axisymmetric oblate bodies (Lambas, Maddox \& Loveday 1992), although lop-sided systems are not rare. The ellipticity of dwarfs was more recently studied by Binggeli \& Popescu (1995) for Virgo cluster dwarfs and Sung et al. (1998) for a sample of field UGC selected dwarfs. While the number of gas-rich dwarfs is low in a rich cluster environment, Binggeli \& Popescu found that Im/dI type dwarfs were slightly rounder than late-type spirals. The Sung sample was selected by morphological criteria, similar to the types of dwarfs used on our sample, and they conclude that dwarfs and disks divide into structurally distinct populations. Late-type disks are found to be oblate spheroids with axial ratios of 1:1:0.2, whereas, dwarf irregulars are triaxial with mean axial ratios of 1:0.7:0.5. However, even in the extreme case of spherical dwarfs and oblate disks, this would only account for an increase into $2 \mathrm{D}$ scale length of a factor of 1.2. The correlations in Figure 2 are separated by a factor of 2.5, which cannot be explained by a mere shift in the shape of dwarfs.

\section{DISCUSSION}

\subsection{Morphological Differences Between Disks and Dwarfs}

The two sequences in Figure 2 provide a new view on galaxy type outside the usual morphological definitions. While, in the last section, we have referred to the two sequences as dwarf and disk, this is only because of the fact that a majority of the dwarf galaxies lie on one sequence and a majority of the disk galaxies lie on the other, where we take the general definition of a dwarf as a small, low mass 
system and a disk is a oblate, rotating galaxy. There is a small, but significant, overlap in physical parameters such luminosity (mass), size (scale length) and mass density (surface brightness) between the two sequences. Therefore, there is no boundary of mass, size or density to classify a galaxy as a dwarf without applying an arbitrary division (Sandage \& Binggeli 1984). In fact, the greatest importance for the two sequences in the $M_{*}-\alpha$ plane is that it allows for a new technique to classify galaxies as either dwarf or disk, based on two physical parameters (luminosity and scale length).

Since the distinction used herein between dwarf and disk in Figures 1 and 2 is based strictly on morphological appearance of the galaxy, and not any characteristic as defined by mass or kinematics, it is worth examining the morphological characteristics of the three samples. The Courteau and de Jong samples use the Hubble system to select a set of disk galaxies. For Courteau, this is a sample of Sc galaxies that are, to a high probability, a uniform set of rotating oblate spheroids with small bulges and a clear spiral pattern. The sample from de Jong covers a wider range of Hubble classes, selected to be undisturbed and, therefore, also forms a subset of the best examples of their morphological classes. The late-type galaxies in all three samples can be broken down into three morphological types; those with bulges and LSB disks (Sc and Sd class), those with apparent axial symmetry but no bulges (Sm class) and those with no symmetry (Im and dI class). The de Jong and Courteau samples contain only those galaxies with axial symmetry (Sa to Sm), so a disk galaxy in Figures 1 and 2 refers to this morphological definition.

The dwarf sample was also selected on morphological grounds and focuses on Im and dI class objects (although it also contains a number of Sm type systems, see Schombert, Pildis \& Eder 1997). We found in a previous paper (Eder \& Schombert 2000) that an optimal technique to produce a sample of low mass, gas-rich, small-sized galaxies was to search for LSB objects with irregular morphology (the goal was to map the large scale structure of dwarfs). However, that technique does not preclude the inclusion of LSB disks into the sample. For example, there are several examples of Sm type galaxies in the LSB dwarf catalog that are neither low in stellar mass (luminosity) or small in scale length, but have sufficiently irregular morphologies to appear similar to dwarf irregulars. The rationale for the use of irregular morphology to locate dwarf galaxies is a practical one. Low mass density implies irregularity, at least with respect to the pattern of star formation (Sneiden \& Gerola 1979), thus irregular structure is nominally associated with dwarf galaxies (unless the star formation is completed, as in a dwarf elliptical). Other sources of irregular morphology, such as galaxy-galaxy interactions, are normally associated with strong star formation activity and other tidal signatures. Of course, irregular morphology by itself is not an exact predictor of low mass for there are many irregular galaxies that are massive (Hunter \& Gallagher 1986). Likewise, axial symmetry does not immediate guarantee that a galaxy is massive as there are many spirals that are dwarf-like in luminosity and size (Schombert et al. 1995).

The three samples provide a range of Hubble classes, with overlap in the Sm class between the LSB dwarfs and de Jong's sample. All the galaxies classified as Im or dI lie on the dwarf sequence and all the galaxies classified from Sa to Sd lie on the disk sequence regardless of the sample from which they are derived. The transition objects, the Sm class, all fall on the dwarf sequence regardless if they are from the de Jong sample or the LSB dwarf sample. This is surprising since while Sm's are irregular, they are not chaotic and are certainly disk systems in terms of being oblate spheroids (Sandage \& Binggeli 1984, Sandage, Binggeli \& Tammann 1985). This indicates that something more fundamental than just disk-like shape and rotation underlies the division found in Figure 2. 
We also note that the two sequences appear as one continuous sequence when H I mass versus scale length is considered (right panel in Figure 2). This may be because of the fact that an optical measure of size is being compared to the gas mass (H I flux). Dwarf galaxies are known to be more extended in H I compared to their optical image (van Zee, Haynes \& Giovanelli 1995). If the scale length $(\alpha)$ was determined from H I images for these galaxies, it seems plausible that those values would be larger and the two sequences would again be distinguishable. Carignan et al. (1990) find that the typical H I extent of a dwarf irregular is twice to three times that of its optical extent, while spirals are only $50 \%$ larger in HI. If spirals have smaller H I distributions than dwarfs, then this would account for the lack of separation for H I mass versus scale length in Figure 2.

\subsection{Kinematic Differences Between Disks and Dwarfs}

The underlying reason to the structural differences between dwarfs and disks may be due to kinematic differences. A first estimate of the kinematics in gas-rich galaxies derives from the shape of their H I line profiles. These profiles can be divided into two types, gaussian and double-horned. A galaxy with a flat or rising rotation curve, and a distribution of gas that declines with radius in the form of a power law, will exhibit a H I profile that has two peaks with a flat plateau region between them, the so-called double-horned profile (see Giovanelli \& Haynes 1988). Those galaxies lacking in rotation (or observed face-on) will display a gaussian profile where its width reflects the internal velocity dispersion of the system.

All late-type spirals (except for face-on systems) have double-horned profiles since they are oblate rotators. However, there is no a priori reason to believe that an irregular dwarf sample would also be composed solely of double-horned profiles. For example, their irregular shape may be due to the distribution of gas and stars determined by pressure support from an anisotropic velocity distribution. To test for the frequency of rotation in LSB dwarfs, we examined the H I profiles of the galaxies in the Eder \& Schombert sample. Of the 106 galaxies in their sample, 67 (63\%) display clear double-horned H I profiles. However, half of the remaining 39 galaxies have profiles with line widths less than 50 $\mathrm{km} / \mathrm{sec}$. A profile width this narrow is impossible to distinguish between gaussian or double-horned at the resolution used on Arecibo system. Therefore, their classification is unknown. The remaining 20 galaxies have resolved gaussian shaped profiles, but this is only $24 \%$ of the total sample. If dwarfs are nearly oblate, then this value is consistent with the remaining fraction of profiles being gaussian because of their nearly face-on orientations to the Earth. Thus, we conclude that a majority of LSB dwarfs have kinematics were rotation is evident and it is plausible that all LSB dwarfs have rotation kinematics. This also agrees with high resolution H I mapping of late-type galaxies (Swaters et al. 2002) who finds that, although their rotation curves are flatter than spirals, there is no indication that dwarf galaxies are not rotation supported.

This means that the separation between dwarfs and disks seen in Figure 2 is not due to a simple shift in the internal kinematics from rotation to non-rotation, at least not with respect to the kinematics of the gas. The stars may follow different kinematics (Hunter et al. 2002) although the gas and stars have similar density distributions in LSB galaxies (McGaugh, Schombert \& Bothun 1995). However, there is one key difference between the rotation of dwarfs and that of spiral disks, the rotation values for dwarfs are much lower than spirals. In this low rotation realm, turbulent motion can compete with 
rotation as the primary influence on a galaxy's kinematics (Seiden \& Gerola 1979). As turbulent motion increases, relative to rotation, the structure of a galaxy begins to deviate from an oblate spheroid. A triaxial structure, as indicated by the Sung et al. (1998) study, then forms where the shape of a galaxy begins to take on the a global structure influenced by a slightly anisotropic velocity distribution. It is possible that this change, from rotation dominance to a stronger turbulent component, in the kinematics of dwarfs is reflecting into structure and is signaled by the two sequences in the $M_{*}-\alpha$ diagram. Unfortunately, this scenario provides no explanation for why there are two distinct sequences rather than a smooth transition in structure from an oblate shape to a triaxial one as one progresses to lower galaxy mass.

A higher ratio of turbulent motion to rotation would also explain the irregular morphology of dwarfs. In disk galaxies, the symmetric nature to rotation reflects in the axial symmetric luminosity distribution, broken only by local regions of higher surface brightness star formation. The flat rotation curve produces the star formation patterns that define the Hubble sequence. However, H I mapping of dwarfs shows that their kinematics follow solid body rotation (van Zee et al. 1997). With solid body rotation dominating over most of the optical region of a dwarf galaxy, random cloud motion can produce a chaotic luminosity distribution rather than a spiral pattern. In this sense, our results follow the scenario proposed by Brosch, Heller \& Almoznino (1998) where the irregular morphology in dwarfs is not caused by stochastic star formation, but rather, the irregular structure is because of an asymmetry in the dark matter halo and reflecting an irregular kinematic distribution.

\subsection{Star Formation in Dwarfs and Disks}

In Schombert, McGaugh \& Eder (2001), we showed that the stellar mass to gas mass relation for

spiral disks is steeper than for LSB dwarfs. A steeper correlation for disk galaxies implies that they have been more efficient at converting gas into stars in the past. This also agrees with the observation that disk galaxies typically have a greater amount of their baryonic mass in stars rather than gas (McGaugh $\&$ de Blok 1997) and that dwarf galaxies have young spectral signatures $\left(D_{n}(4000)\right.$ and $\mathrm{H} \beta$, Kauffmann et al. 2003) .

One possible interpretation to the change in the relationship between gas and stellar mass in dwarfs and disks is to consider the effects that different kinematics, suggested in the last section, has on star formation processes. Star formation mechanisms are, basically, methods of increasing local gas densities until the Jeans mass is reached. At this point, fragmentation occurs followed by collapse into protostars. There are three primary methods to achieve this state; 1 ) cloud collisions (Elmegreen 1990, Scoville et al. 1991), 2) density waves (Kennicutt 1998) and 3) induced by shocks from SN explosions or stellar winds (Elmegreen \& Parravano 1994).

Considering cloud collisions first, the formation of clouds in disk systems is primarily confined to the plane of the galaxy. Since rotation is dominant, clouds will tend to gently merge (i.e. with velocities similar to their internal velocities) with other clouds on the similar orbits, but with slightly different orbital eccentricities. If in dwarfs, on the other hand, clouds are dispersed in a more diffuse, triaxial environment under the influence of turbulent motion, then the cross section for collisions is smaller in this environment and, when they do collide, the collisions will tend to be head-on and serve to dissipate 
the clouds rather than increase their densities. Thus, star formation, averaged over the lifetime of a galaxy, will be less efficient in dwarf galaxies as compared to disks.

Density waves in disk galaxies also serve to focus star formation events. Clumping of molecular clouds has been noted in several spiral galaxies (Vogel, Kulkarni \& Scoville 1988, Rand 1993, Vogel et al. 1993), all of which lead to enhanced star formation. In particular, this style of star formation becomes an orderly function of the kinematics of the spiral, and gas is converted into stars in an even, efficient manner. In contrast, the velocity distributions in dwarf galaxies may prevent the build-up of density wave patterns, and star formation becomes a strictly local phenomenon (Hunter, Elmegreen \& Baker 1998) and local star formation is a chaotic process, i.e. less efficient in converting the gas supply into stars.

For induced, stochastic star formation, the mechanism that triggers cloud collapse is a superbubble shock wave. The shock waves arise from a combination of stellar winds originating in young clusters and/or supernova explosions (Tenorio-Tagle \& Bodenheimer 1988, Miller \& Scalo 1978). In disk

galaxies, pattern speed is important because if a cloud spends more than $10^{7}$ yrs in arm region then the chances that a nearby explosion will trigger star formation are high (Sleath \& Alexander 1996). If there is a larger component of random motion in dwarfs, than this lowers the probability that a cloud will be located near an explosion, and therefore lowers the star formation efficiency compared to a disk galaxy with coherent motion. $\mathrm{H} \alpha$ and $\mathrm{H}$ I maps of dwarfs confirm this chaotic aspect to star formation in dwarfs (Walter \& Brinks 1999).

In summary, regardless of the exact mechanism for star formation, it is clear that the rotation of disk galaxies leads to a more coherent star formation pattern and more efficient conversion of gas into stars. In contrast, if LSB dwarf galaxies have a larger component of random motion over ordered rotation, then they will lack any coherent structure other than that temporarily provided by self-progating star formation. This, in turn, leads to random star formation and inefficient use of the gas supply. The end result is a galaxy that has distributed its stars over a wider area (i.e. more diffuse as shown in Figure 2) versus a spiral galaxy that has focused a majority of their stars in the compact disk region.

\subsection{Dwarf Galaxy Formation}

Figure 2 indicates that, per unit of stellar mass, dwarf galaxies have larger scale lengths than disk galaxies. If both types of galaxies are oblate rotators, and a majority of their stellar mass is in the shape of a flattened spheroid component, then the differences detected in Figure 2 would indicate that dwarfs and disks follow a different scaling law that describes how stellar matter is distributed in their gravitational wells. Dwarf galaxies, as a class, have more of their stellar material at larger radii compared to disk galaxies of the same mass, which also produces lower central stellar densities (see Figure 1). It is important to note that this difference is not a continuous sequence in stellar mass as there are clearly two distinct relationships for dwarfs and disks in Figure 2. Thus, despite whatever morphological criteria has led us to classify dwarfs and disk galaxies, there is also some underlying difference in the way stellar matter traces the gravitational potential that separates the two types of galaxies. Dwarf galaxies are not simply scaled down versions of disk galaxies, although the stellar light distribution in both types follows an exponential law. These structural differences may have been 
imposed at the time of galaxy formation. For example, all galaxies have substantial dark matter halos, but the ratio of dark to luminous matter is particular high in LSB dwarfs (de Blok \& McGaugh 1997). Thus, the dwarf and disk sequences may represent two families of galaxies that follow different formation paths or different coupling methods to the dark matter halos in which they reside.

Aside from morphology, gas-rich galaxies display a large degree of regularity in their global properties (Roberts \& Haynes 1994). The recent advent of large galaxy datasets (i.e. Sloan Digital Sky Survey, SDSS) allows a broader comparison of galaxy characteristics, and in quantitative ways. Several recent studies have noted a difference in galaxy properties (such as spectral and concentration indices) between high and low mass systems (Tremonti et al. 2004, Kauffmann et al. 2003). Low mass galaxies in the SDSS samples display younger stellar populations, lower surface mass densities and lower mean concentrations (i.e. lower mean surface brightnesses) than high mass galaxies. The division between the two families occurs at $3 \times 10^{10} M_{\odot}$, which is effectively the upper limit of the dwarf sequence as seen in Figure 2. Their correlations between stellar mass and various spectral indices also overlap in the region of $5 \times 10^{9} M_{\odot}$ to $5 \times 10^{10} M_{\odot}$, identical to the overlap region between disk and dwarfs herein.

Structurally, the Kauffmann et al. SDSS study finds a continuous sequence from high to low mass galaxies where the concentration index for low mass galaxies is lower than high mass galaxies (more diffuse), but the distinction is not as clear as their spectral indices because of their use of a mean surface brightness value rather a direct profile fit scale length (i.e. $\alpha$ ). In contrast, the results presented here demonstrate that our two families (disk and dwarfs) overlap in size, but form two distinct sequences in the $M_{*}-\alpha$ plane. We also note that the distinction between the disk and dwarf sequences is less noticeable when only the gas mass is considered (right panel of Figure 2), but this is a comparison between the optical scale length $(\alpha)$ and the H I gas mass. Since the distribution of H I gas in dwarfs is typically more extended than the optical light (van Zee, Haynes \& Giovanelli 1995), then it is reasonable to assume that the two sequences would be clearer when using H I scale length and, also, the total baryonic mass (stellar plus gas mass).

In addition to star formation history and structural differences, low mass galaxies also display different mass-metallicity relations as compared to high mass systems (Garnett 2002). Garnett's work finds a uniform change in $O / H$ abundance with mass for galaxies with rotational velocities $\left(V_{\text {rot }}\right)$ greater than $125 \mathrm{~km} / \mathrm{sec}$. Lower mass galaxies have a steeper mass-metallicity slope that is interpreted as an increased loss of metals from low mass galaxies by galactic winds. It will be a challenge to galaxy formation models to explain why there are strictly two sequences. One solution would be the hierarchical clustering and merging scenario of galaxy formation (Kauffmann, White \& Guiderdoni 1993, Kauffmann \& Charlot 1998) where the disk sequence is built by accertion from dwarf galaxies. However, this would mean that the dwarfs are primordial, which is not true of a majority of their stellar populations.

\section{CONCLUSIONS}

The primary goal of this paper is to demonstrate that there is a clear distinction between standard disk galaxies and irregular dwarf galaxies in the mass (luminosity) versus scale length $(\alpha)$ plane. Whether a galaxy lies on the dwarf sequence is not solely determined by either morphology, luminosity,

size, density or kinematics. Yet the combination of stellar mass and scale length produces two sequences 
that are distinct and must reflect some underlying physics that is unique to dwarf galaxies as compared to spirals. In fact, the distinction between the two sequences is clear enough to serve as a future definition of a dwarf galaxy (as one on the upper sequence) and a disk galaxy (as one on the lower sequence) regardless of appearance.

We summarize our conclusions as the following:

(1) Even though there is no single optical characteristic that distinguishes dwarfs from disk galaxies, both types are clearly separated in the $M_{*}-\alpha$ diagram (Figure 2). Given that the Tully-Fisher relation for these two samples of dwarfs and disks produces an extremely tight relation between baryonic mass and rotational velocity (McGaugh et al. 2000), the difference must be in how the scale length develops in dwarfs rather than any function of mass (either stellar or gas). Therefore, we conclude that the dwarf galaxies form a distinct sequence that is more diffuse (larger scale length, $\alpha$, for its mass) than disk galaxies. The disk galaxies define a similar linear sequence despite the vast range in Hubble type and central surface brightness, $\mu_{o}$.

(2) While the morphological separation is not perfect in Figure 2, all Hubble types from Sc to Sd lie on the disk sequence and all Hubble types Im and dI lie on the dwarf sequence. A majority of the class Sm galaxies lie on the dwarf sequence, despite their apparent oblate, disk shape.

(3) Several recent studies of the SDSS dataset have noted a difference in galaxy properties (such as spectral and concentration indices) between high and low mass systems (Tremonti et al. 2004, Kauffmann et al. 2003). The division between the two families occurs at $3 \times 10^{10} M_{\odot}$, which is effectively the upper limit of the dwarf sequence as seen in Figure 2. The existence of two families of galaxies would support hierarchical clustering and merging scenarios of galaxy formation (Kauffmann, White \& Guiderdoni 1993, Kauffmann \& Charlot 1998) where the dwarf sequence evolves into the disk sequence by accretion.

(4) The structural difference between dwarfs and disks may reflect small shape and kinematic differences between the two populations. If the stellar and gas distribution of dwarfs are triaxial (Sung et al. 1998), then the only quantitative difference between the kinematics of dwarfs and disks is the ratio of their large-scale turbulent motion to rotation, where dwarfs have low rotation rates and stronger non-axial motion. This would lead to a more diffuse stellar distribution and irregular morphology.

(5) The importance of large-scale turbulent motion over rotation would also explain the irregular star formation patterns in dwarf galaxies. This would also disrupt the internal star formation processes and explain the why dwarfs have been more inefficient at converting gas mass into stellar mass. However, this hypothesis is tentative as we would expect a smooth transition from low mass dwarfs to higher mass disks. Instead, the two sequences signal two distinct paths of galaxy evolution, perhaps controlled by separate processes.

Existence of two families of gas-rich galaxies suggests that young galaxies follow at least two paths during their primeval stage after collapse of the initial lump of dark and baryonic material. For example, high mass galaxies can dissipate and, by conservation of angular momentum, become rotating disk galaxies. What turbulent motion was contained in the original protogalaxy is overwhelmed by 
dissipation into the ordered motion of rotation. For low mass lumps, rotational kinematics still dominate, but large-scale turbulent motion plays a minor, but significant, role by slowing the collapse that, in turn, leads to a more diffuse stellar distribution and a primeval dwarf galaxy with chaotic structure. Thus, morphology follows from a combination of internal kinematics and star formation history. Likewise, as proposed by hierarchical models, dwarf galaxies form first as irregular structures than merge into spiral disks where dissipation introduces ordered motion and star formation.

The feedback from the kinematics to star formation becomes one of the relative importance of ordered motion over disordered motions. Ordered motion, as found in disks, leads to higher star formation rates and more efficient conversion of gas into stars. This efficient style of star formation also feedbacks into the development of patterns and density waves, which guides the morphological appearance of spirals. Thus, the highly efficient disk systems develop into early-type spirals with high star formation rates, high central surface brightnesses and a rapid use of their gas supplies. Less ordered systems become the late-type disk galaxies with more fragmented morphological appearance dominated by bursts of star formation and early baryonic blowout of metal enriched gas. This scenario finds support from estimates of the gas depletion timescales for spirals, which are less than a Hubble time but greater than that for dwarfs (Schombert, McGaugh \& Eder 2001).

At the far end of this scheme lie the LSB dwarfs. While they have rotation kinematics, this rotation is primarily solid-body and, thus, there will be fewer cloud collisions that induces cooling plus fewer patterns of density for gas to collect and less time spent in the regions of shocks waves from recent star formation, which would induce new star formation. Based on the current low surface brightness nature of the stellar population, this inhibited star formation history would stretch all the way back to the formation of the dwarf. While this scenario emphasizes the influence of internal factors on the evolution of a dwarf, environmental factors would clearly have an enormous impact, especially in clusters; however, this cannot be tested for in the current LSB dwarf sample as it is a pure field sample (Schombert et al. 1997).

We wish to thank the generous support of the Arecibo Observatory for the allocation of time to search for HI emission from the candidate dwarf galaxies and Michigan- Dartmouth-M.I.T Observatory in carrying out the photometry portion of this program This work is based on photographic plates obtained at the Palomar Observatory 48-inch Oschin Telescope for the Second Palomar Observatory Sky Survey, which was funded by the Eastman Kodak Company, the National Geographic Society, the Samuel Oschin Foundation, the Alfred Sloan Foundation, the National Science Foundation and the National Aeronautics and Space Administration.

\section{REFERENCES}

Bell, E. \& de Jong, R. 2001, ApJ, 550, 212

Binggeli, B. \& Popescu, C. 1995, A\&A, 298, 63

Brosch, N., Heller, A. \& Almoznino, E. 1998, MNRAS, 300, 1091

Carignan, C., Charbonneau, P., Boulanger, F. \& Viallefond, F. 1990, A\&A, 234, 43

Courteau, S. 1996, ApJS, 103, 363 
Dalcanton, J., Spergel, D. \& Summers, F. 1997, ApJ, 482, 659

de Blok, W. \& McGaugh, S. 1997, MNRAS, 290, 533

Eder, J. \& Schombert, J. 2000, ApJS, 131, 47

Elmegreen, B. 1990, ApJ, 357, 125

Elmegreen, B. \& Parravano, A. 1994, ApJ, 435, L121

Fall, S. \& Efstathiou, G. 1980, MNRAS, 193, 189

Garnett, D. 2002, ApJ, 581, 1019

Giovanelli, R. \& Haynes, M. 1988, in Galactic and Extragalactic Radio Astronomy, (New York:SpringerVerlag), p. 522

Graham, A. \& Driver, S. 2005, PASA, 22, 118

Hoffman, G., Dickey, J., Lu, N. \& Fromhold-Treu, R. 1996, ApJ, 473, 822

Hunter, D., Elmegreen, B. \& Baker, A. 1998, ApJ, 493, 595

Hunter, D. \& Gallagher, J. 1986, PASP, 98, 5

Hunter, D., Rubin, V., Swaters, R., Sparke, L. \& Levine, S. 2002, ApJ, 580, 194

Kauffmann, G., Heckman, T., White, S., Charlot, S., Tremonti, C., Peng, E., Seibert, M., Brinkmann, J., Nichol, R., SubbaRao, M. \& York, D. 2003, MNRAS, 341, 54

Kauffmann, G. \& Charlot, S. 1998, MNRAS, 294, 705

Kauffmann, G., White, S. \& Guiderdoni, B. 1993, MNRAS, 254, 201

Kennicutt, R. 1998, ApJ, 498, 541

Lambas, D., Maddox, S. \& Loveday, J. 1992, MNRAS, 258, 404

McGaugh, S., Schombert, J. \& Bothun, G. 1995, AJ, 109, 2019

McGaugh, S. \& de Blok, W. 1997, ApJ, 481, 689

McGaugh, S., Schombert, J., Bothun, G. \& de Blok, W. 2000, ApJ, 533, L99

Miller, G. \& Scalo, J. 1978, PASP, 90, 506

Papaderos, P., Loose, H., Fricke, K. \& Thuan, T. 1996, A\&A, 314, 59

Patterson, R. \& Thuan, T. 1996, ApJS, 107, 103

Pildis, R., Schombert, J. \& Eder, J. 1997, ApJ, 481, 157

Rand, R. 1993, ApJ, 410, 68

Roberts, M. \& Haynes, M. 1994, ARA\&A, 32, 115

Sandage, A., Binggeli, B. \& Tammann, G. 1985, AJ, 90, 395

Sandage, A. \& Binggeli, B. 1984, AJ, 89, 919

Schombert, J., McGaugh, S. \& Eder, J. 2001, AJ, 121, 2420

Schombert, J., Pildis, R. \& Eder, J. 1997, ApJS, 111, 233

Schombert, J., Pildis, R., Eder, J. \& Oemler, A. 1995, AJ, 110, 2067

Scoville, N., Sargent, A., Sanders, D. \& Soifer, B. 1991, ApJ, 366, L5 
Swaters, R., van Albada, T., van der Hulst, J. \& Sancisi, R. 2002, A\&A, 390, 829

Sleath, J. \& Alexander, P. 1996, MNRAS, 283, 358

Seiden, P. \& Gerola, H. 1979, ApJ, 223, 56

Sung, E., Han, C., Ryden, B., Patterson, R., Chun, M., Kim, H. \& Kim, D. 1998, ApJ, 505, 199

Tenorio-Tagle, G. \& Bodenheimer, P. 1988, ARA\&A, 26, 145

Tremonti, C., Heckman, T., Kauffmann, G., Brinchmann, J., Charlot, S., White, S., Seibert, M., Peng, E., Schlegel, D., Uomoto, A., Fukugita, M. \& Brinkmann, J. 2004, ApJ, 613, 898

Vogel, S., Kulkarni, S. \& Scoville, N. 1988, Nature, 334, 402

Vogel, S., Rand, R., Gruendl, R. \& Teuben, P. 1993, PASP, 105, 666

Walter, F. \& Brinks, E. 1999, AJ, 118, 273

Worthey, G. 1994, ApJS, 95, 107

de Jong, R. 1996, A\&A, 118, 557

van Zee, L., Haynes, M. \& Giovanelli, R. 1995, AJ, 109, 990

van Zee, L., Haynes, M., Salzer, J. \& Broeils, A. 1997, AJ, 113, 1618

van Zee, L., Salzer, J. \& Skillman, E. 2001, AJ, 122, 121

This preprint was prepared with the AAS LATEX macros v5.2. 